\title{
Colorectal Cancer Prognosis Is Not Associated with $B R A F$ and KRAS Mutations-A STROBE Compliant Study
}

\author{
Joon-Hyop Lee ${ }^{1,+} \mathbb{D}^{\mathbb{C}}$, Jiyoung Ahn ${ }^{2,+}$, Won Seo Park ${ }^{3} \mathbb{D}$, Eun Kyung Choe ${ }^{4}$, Eunyoung Kim ${ }^{5}$, \\ Rumi Shin ${ }^{6}$, Seung Chul Heo ${ }^{6}$, Sohee Jung ${ }^{7}$, Kwangsoo Kim ${ }^{7}$, Young Jun Chai ${ }^{6, * 10}$ and \\ Heejoon Chae ${ }^{2, *(\mathbb{D})}$ \\ 1 Department of Surgery, Gachon University College of Medicine, Gil Medical Center, Incheon 21565, Korea; \\ deftnovice@gmail.com \\ 2 Division of Computer Science, Sookmyung Women's University, Seoul 04310, Korea; jiyoung464@naver.com \\ 3 Department of Surgery, Kyung Hee University Hospital, Seoul 02447, Korea; pwsmd@hanmail.net \\ 4 Department of Surgery, Seoul National University Hospital Healthcare System, Gangnam Center, \\ 101 Daehak-ro, Seoul, Korea; choe523@gmail.com \\ 5 Department of Surgery, National Medical Center, Seoul 04564, Korea; keyss30809@naver.com \\ 6 Department of Surgery, Seoul Metropolitan Government-Seoul National University Boramae Medical \\ Center, Seoul 07061, Korea; roomie79@gmail.com (R.S.); heosc3@brmh.org (S.C.H.) \\ 7 Division of Clinical Bioinformatics, Biomedical Research Institute, Seoul National University Hospital, \\ Seoul 03080, Korea; sh0202j@gmail.com (S.J.); kksoo716@gmail.com (K.K.) \\ * Correspondence: kevinjoon@naver.com (Y.J.C.); heechae@sookmyung.ac.kr (H.C.); \\ Tel.: +82-2-870-3296 (Y.J.C.), +82-2-2077-7414 (H.C.) \\ + These authors contributed equally.
}

Received: 26 December 2018; Accepted: 14 January 2019; Published: 17 January 2019

check for updates

\begin{abstract}
Background: We investigated the associations between $\mathrm{v}$-Raf murine sarcoma viral oncogene homolog $\mathrm{B} 1\left(B R A F^{\mathrm{V} 600 \mathrm{E}}\right.$, henceforth $\left.B R A F\right)$ and v-Ki-ras2 Kirsten rat sarcoma viral oncogene homolog (KRAS) mutations and colorectal cancer (CRC) prognosis, using The Cancer Genome Atlas (TCGA) and the Gene Expression Omnibus (GSE39582) datasets. Materials and Methods: The effects of $B R A F$ and KRAS mutations on overall survival (OS) and disease-free survival (DFS) of CRC were evaluated. Results: The mutational status of BRAF and KRAS genes was not associated with overall survival (OS) or DFS of the CRC patients drawn from the TCGA database. The 3-year OS and DFS rates of the BRAF mutation (+) vs. mutation (-) groups were $92.6 \%$ vs. $90.4 \%$ and $79.7 \%$ vs. $68.4 \%$, respectively. The 3 -year OS and DFS rates of the KRAS mutation (+) vs. mutation (-) groups were $90.4 \%$ vs. $90.5 \%$ and $65.3 \%$ vs. $73.5 \%$, respectively. In stage II patients, however, the 3 -year OS rate was lower in the BRAF mutation (+) group than in the mutation $(-)$ group $(85.5 \%$ vs. $97.7 \%, p<0.001)$. The mutational status of $B R A F$ genes of 497 CRC patients drawn from the GSE39582 database was not associated with OS or DFS. The 3-year OS and DFS rates of BRAF mutation (+) vs. mutation (-) groups were $75.7 \%$ vs. $78.9 \%$ and $73.6 \%$ vs. $71.1 \%$, respectively. However, KRAS mutational status had an effect on 3-year OS rate $(71.9 \%$ mutation $(+)$ vs. $83 \%$ mutation $(-), p=0.05)$ and DFS rate $(66.3 \%$ mutation $(+)$ vs. $74.6 \%$ mutation $(-), p=0.013)$. Conclusions: We found no consistent association between the mutational status of BRAF nor KRAS and the OS and DFS of CRC patients from the TCGA and GSE39582 databases. Studies with longer-term records and larger patient numbers may be necessary to expound the influence of $B R A F$ and $K R A S$ mutations on the outcomes of CRC.
\end{abstract}

Keywords: colorectal cancer; $B R A F$; KRAS; overall survival; disease-free survival 


\section{Introduction}

Colorectal cancer (CRC) is one of the most common cancers worldwide, representing the second most common form of cancer diagnosed in females and the third most common form of cancer diagnosed in males [1]. Although the decreasing mortality rate is attributed to early detection and treatment advances, CRC currently ranks fourth for cancer-related mortality [2]. The TNM stage, a staging system defined by the American Joint Committee on Cancer (AJCC) based on pathologic and clinical factors, is the conventional parameter by which CRC prognosis and treatment is determined [3].

Research into the molecular and genetic mechanisms of CRC carcinogenesis and progression in recent decades prompted the search for genetic prognostic factors to complement the TNM staging system for all malignancies. Public availability of genomic databases such as The Cancer Genome Atlas (TCGA) and the Gene Expression Omnibus (GEO, GSE39582) dataset now allows clinicians and bioinformatics researchers to conduct genomic research on different cancers, including CRC, which has not generally been possible in the past [4-7]. There are several different molecular and genomic mechanisms that may contribute to CRC formation such as microsatellite instability phenotype, CpG island methylator phenotype [8], and chromosomal instability [9].

Research and systematic reviews have primarily focused on v-Raf murine sarcoma viral oncogene homolog B1 (BRAF) and v-Ki-ras2 Kirsten rat sarcoma viral oncogene homolog (KRAS) genes as potential prognostic biomarkers for CRC. Despite conflicting reported effects of $B R A F$ and $K R A S$ mutations [10-13] the presence of either are additional factors recommended in the 8th Edition of the AJCC guidelines for influencing clinical care $[3,11,14]$. In the present study we assessed the association between $B R A F^{\mathrm{V} 600 \mathrm{E}}(B R A F)$ and KRAS mutations and the outcome of CRC patients by TNM stage using the publicly available TCGA and GSE39582 datasets.

\section{Materials and Methods}

\subsection{Data Acquisition and Selection}

The data of 536 patients with genomic variant colon adenocarcinoma (COAD) and rectal adenocarcinoma (READ) with corresponding clinical information were obtained from the TCGA GDC portal (https://portal.gdc.cancer.gov/). Their mRNA-Seq data were produced with the Illumina HiSeq 2000 (Illumina Inc, CA, USA) platform and processed by the RNAseqV2 pipeline, using MapSplice, v.1.15.2 (University of Kentucky, Lexington, KY, USA) and RSE, v. 1.3.0 for alignment and quantification. After excluding 89 patients with incomplete data (five with lack of clinical information, 69 missing survival data, and 15 lacking CRC stage information), the remaining 447 patients' data were analyzed. In addition, the GSE39582 data of 585 patients were downloaded from the GEO repository (https:/ / www.ncbi.nlm.nih.gov/geo/) [15]. Gene expression profiles were assessed with the Affymetrix U133 Plus 2.0 chip (Thermo Fischer Scientific, MA, USA). Eighty-eight patients were excluded because of incomplete data (40 missing KRAS mutational status, 35 missing BRAF mutational status, 4 missing tumor stage information, and 9 missing survival data). The remaining 497 patients' data were analyzed in the same manner as the TCGA data. The median record length duration (henceforth follow-up period) was described in months with range information. CRC was staged according to the 8th American Joint Committee on Cancer's TNM stages guidelines [3]. Sub-stages were omitted to simplify clinical data.

According to TCGA publication guidelines, there are no restrictions on the publication of these somatic mutation and mRNA sequencing data and no specific permission is required for investigators to publish papers containing or referring to these data (http:/ / cancergenome.nih.gov/publications / publicationguidelines). Furthermore, because IRB approval is not acquired for use of public datasets, our study (which used the TCGA and GEO public databases) was not submitted for such review. The manuscript was written in accordance to the Strengthening the reporting of Observational Studies in Epidemiology protocol [16]. 


\subsection{Definition of BRAF and KRAS Mutation Status}

For the TCGA dataset, we categorized BRAF or KRAS mutations based on information provided by the Mutation Annotation Format files of the Mu-tect caller [17]. The mRNA-Seq data were generated using the Illumina HiSeq 2000 platform and processed by the RNAseqV2 pipeline. Patients were classified positive or negative by their mutation status. In the GSE39582 series, information on the gene expression profiles of the BRAF and KRAS genes was determined by the Affymetrix U133 Plus 2.0 chips. Those who were indicated as " $\mathrm{M}$ " were classified in the mutation (+) groups and those showing "WT" were categorized in the mutation (-) groups.

\subsection{Kaplan-Meier Survival Analysis of BRAF and KRAS Mutations}

The potential influence of BRAF and KRAS mutations on each TNM stage on 3-year patient overall survival (OS) and disease-free survival (DFS) was analyzed using the life table method. The TCGA and the GSE39582 dataset provided clinical information on OS and DFS. Kaplan-Meier estimates were calculated for all possible combination of BRAF and KRAS mutation over each TNM stage. The estimation was calculated by a survival package [18] and plotted by the ggplot2 package [19] in $R$ (version 3.4.2) [20]. Null hypotheses of no difference were rejected if $p$-values were less than 0.05 , or, equivalently, if the $95 \%$ confidence intervals of risk point estimates excluded 1.

\section{Results}

There were 627 CRC patients in the sample that we drew from the TCGA dataset, among which 180 were excluded from analysis due to missing values. From the remaining 447 patients, $83(18.7 \%)$ were stage I, $172(38.4 \%)$ were stage II, $134(30.0 \%)$ were stage III, and $58(12.8 \%)$ were stage IV. In the TCGA database, the median follow-up period of BRAF mutation (+) patients was $22.04(0-133.55)$, for BRAF mutation (-) patients the median follow-up period was $23.98(0-147.90)$, for KRAS mutation (+) patients it was $24.73(0-140.28)$, and for KRAS mutation (-) patients it was 22.04 (0-147.90) months. There were 585 patient data in the GSE39582 dataset, among which 88 were excluded due to missing values. From the remaining 497 patients, 32 (6.4\%) were stage I, $242(48.7 \%)$ were stage II, $163(32.8 \%)$, were stage III, and $60(12.1 \%)$ were stage IV. The median follow-up period of the BRAF mutation (+) patients was $46(1-172)$, for BRAF mutation (-) patients it was $52(0-201)$, for KRAS mutation (+) patients it was 46 (0-201), and for KRAS mutation (-) patients it was 56 (0-192) months (Table 1).

Table 1. Baseline characteristics of datasets.

\begin{tabular}{|c|c|c|c|}
\hline & & TCGA & GSE39582 \\
\hline Total number of patients studied & & 447 & 497 \\
\hline Age at diagnosis (mean $\pm S D$ ), years old & & $65.0 \pm 12.9$ & $66.8 \pm 13.1$ \\
\hline \multicolumn{4}{|l|}{ Gender } \\
\hline & Male & $213(47.7 \%)$ & $271(54.5 \%)$ \\
\hline & Female & $234(52.3 \%)$ & $226(45.5 \%)$ \\
\hline Number of $B R A F$ mutations & & $59(13.2 \%)$ & $49(9.9 \%)$ \\
\hline Number of KRAS mutations & & $384(85.9 \%)$ & $197(39.6 \%)$ \\
\hline \multicolumn{4}{|l|}{ AJCC TNM stage (number of $B R A F / K R A S$ mutations) } \\
\hline & I & $83(10 / 36)$ & $32(1 / 15)$ \\
\hline & II & $172(31 / 77)$ & $242(21 / 83)$ \\
\hline & III & $134(14 / 50)$ & $163(21 / 70)$ \\
\hline & IV & $58(4 / 21)$ & $60(6 / 29)$ \\
\hline \multicolumn{4}{|l|}{ Survival event } \\
\hline & Dead & $46(10.3 \%)$ & $40(8.0 \%)$ \\
\hline & Alive & $404(90.4 \%)$ & $457(92.0 \%)$ \\
\hline Median follow-up time, months (range) & & $23.9(0-147.9)$ & $52(0-201)$ \\
\hline Median time to survival event, months (range) & & $24.4(4.0-99.9)$ & $31(0-183)$ \\
\hline
\end{tabular}

TCGA: The Cancer Genome Atlas; AJCC: American Joint Committee on Cancer; SD: standard deviation; BRAF: v-Raf murine sarcoma viral oncogene homolog B1; KRAS: v-Ki-ras2 Kirsten rat sarcoma viral oncogene homolog. 


\subsection{Individual Effect of BRAF Mutation on Survival Outcome}

In TCGA dataset, there was no significant effect of BRAF mutation on CRC OS and DFS. For the $B R A F$ mutation (+) vs. mutation (-) groups, the 3-year overall survival (OS) rates were $92.6 \%$ vs. $90.4 \%$, respectively ( $p=0.4$, number of patients: 13 vs. 109), and the 3-year disease-free survival (DFS) rates were $79.7 \%$ vs. $68.4 \%$ ( $p=0.47$, number of patients: 11 vs. 84 ), respectively (Figure 1 ). In stage II patients, the 3-year OS rate was lower in the BRAF mutation (+) group than in the mutation (-) group $(85.5 \%$ vs. $97.7 \%, p<0.001$, number of patients: 7 vs. 53$)$. There was no difference in the 3 -year DFS rate of stage II based on the BRAF mutation status $(73.5 \%$ vs. $79.6 \%$ for BRAF mutation (+) vs. mutation (-) groups, $p=0.071$, number of patients: 6 vs. 45) (Figure 2). There were no differences in the 3-year OS or DFS of stage I, III, or IV patients.

(a)
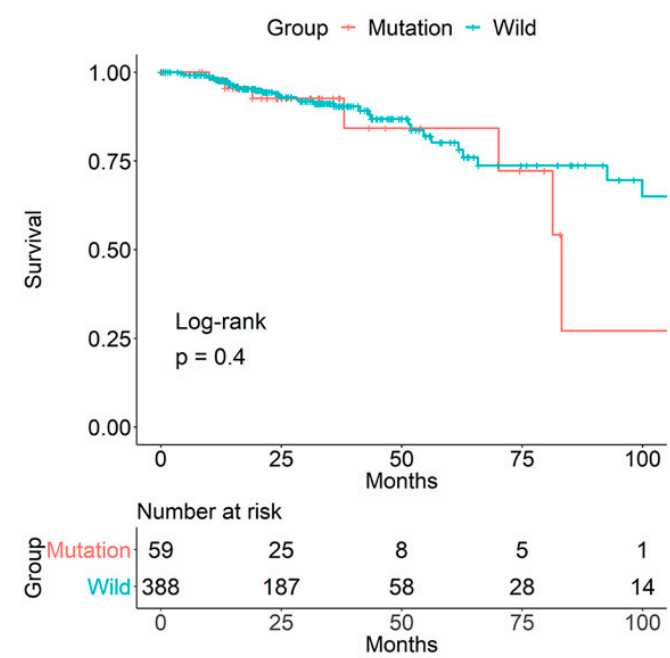

(b)
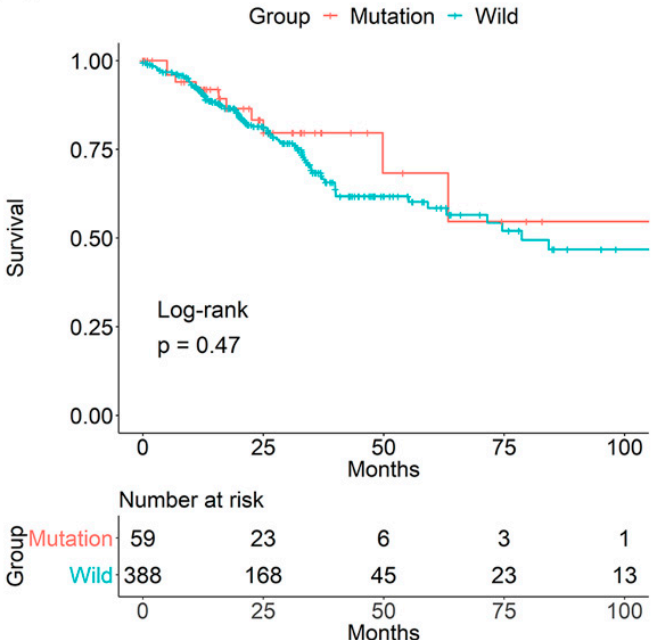

(c)

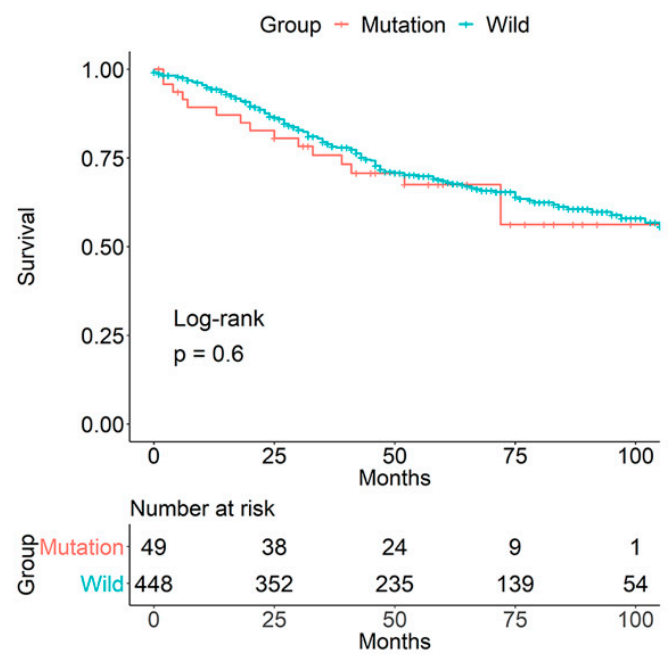

(d)

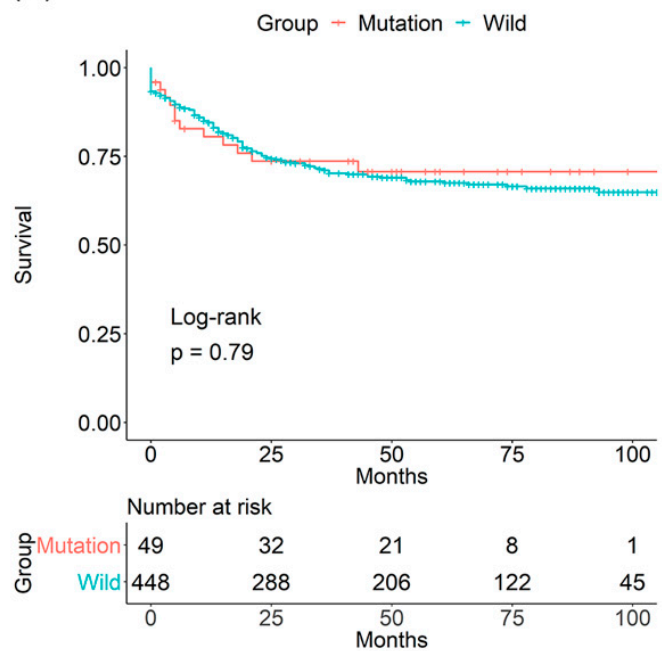

Figure 1. Effect of $B R A F$ mutation on (a) 3-year overall survival and (b) 3-year disease-free survival from the The Cancer Genome Atlas (TCGA) database and on (c) 3-year overall survival and (d) 3-year disease-free survival from the Gene Expression Omnibus (GSE39582) database of colorectal cancer patients of all stages. 
(a)

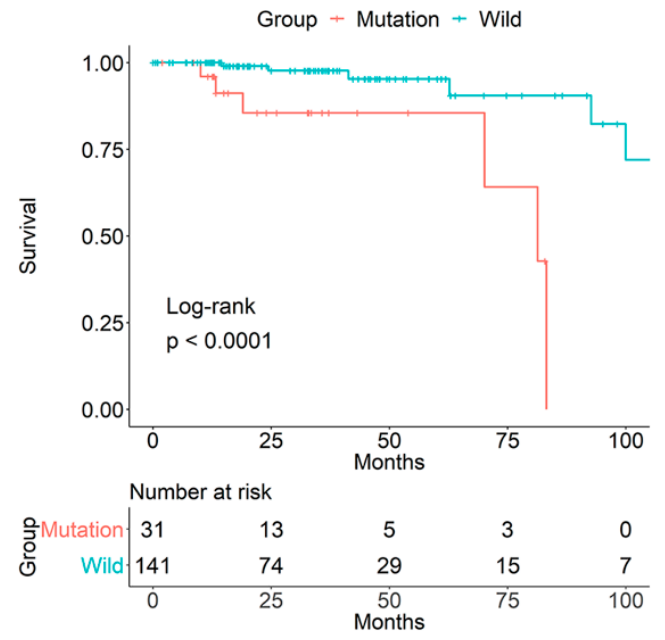

(b)

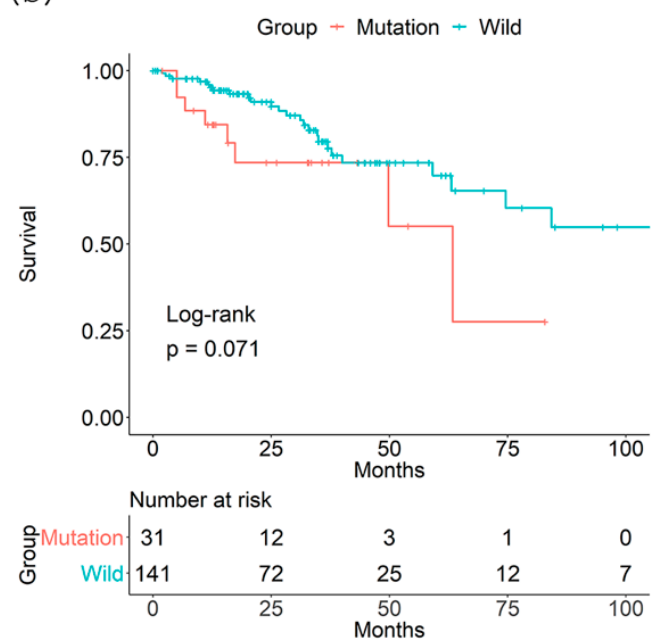

Figure 2. Effect of BRAF mutation on (a) 3-year overall survival and (b) 3-year disease-free survival from the The Cancer Genome Atlas (TCGA) database of colorectal cancer stage II patients.

In the GSE39582 dataset, BRAF mutation status was not associated with the 3-year OS rate $(75.7 \%$ mutation (+) vs. $78.9 \%$, mutation $(-) p=0.6$, number of patients: 30 vs. 300$)$ or DFS rate $(73.6 \%$ mutation (-) vs. $71.1 \%$ mutation $(-), p=0.79$, number of patients: 27 vs. 252) of all CRC patients (Figure 1). The association between $B R A F$ mutation status and 3-year OS for each group from stage I to IV was not significant (data not shown). Regarding 3-year DFS, only the stage III $B R A F$ mutation (+) group (84.4\%) demonstrated a higher 3-year DFS, compared to the mutation (-) group $(62.4 \%$, $p=0.046$, number of patients: 15 vs. 75) (Figure 3). 
(a)

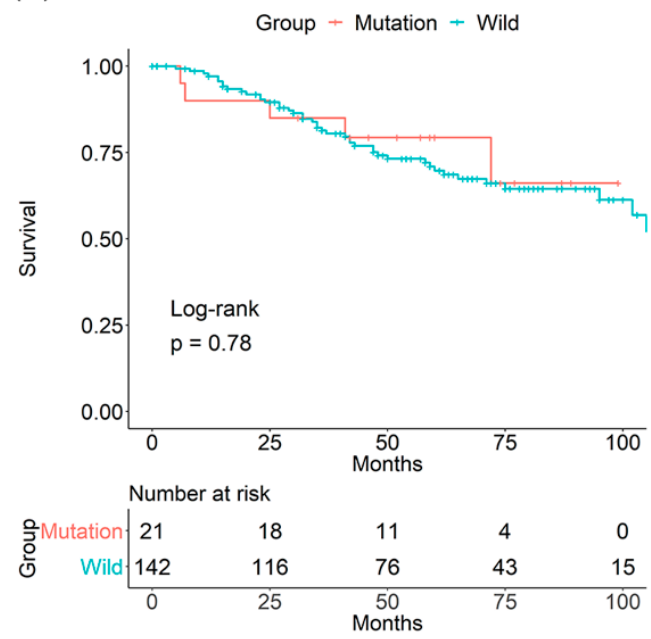

(b)

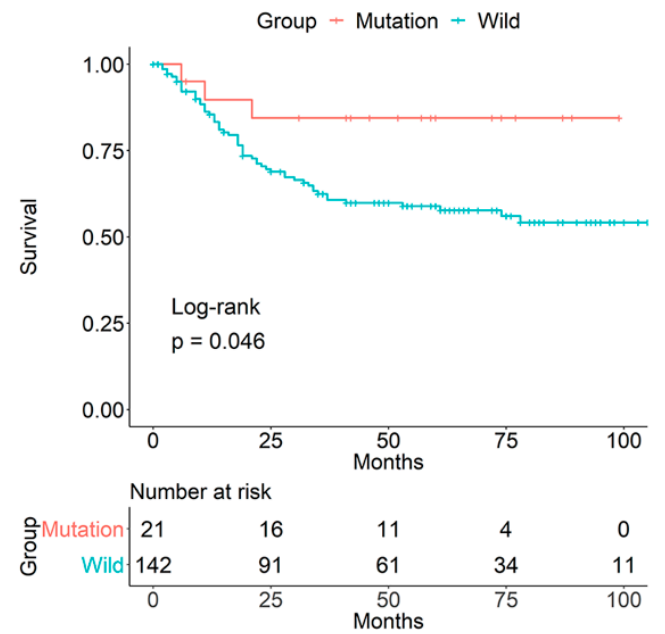

Figure 3. Effect of BRAF mutation on (a) 3-year overall survival and (b) 3-year disease-free survival from the GSE39582 database of colorectal cancer stage III patients.

\subsection{Individual Effect of KRAS Mutation on Survival Outcome}

In the TCGA dataset, there was no significant association between KRAS mutation status and CRC OS or DFS. For KRAS mutation (+) vs. mutation (-) groups, the 3-year OS rates were $90.4 \%$ vs. $90.5 \%$ ( $p=0.64$, number of patients: 61 vs. 61$)$ and the 3-year DFS rates were $65.3 \%$ vs. $73.5 \%(p=$ 0.21 , number of patients: 48 vs. 47 ), respectively (Figure 4). Likewise, the KRAS mutation status was not associated with significant differences across all stages for 3-year OS rates as well as DFS rates (data not shown). 
(a)

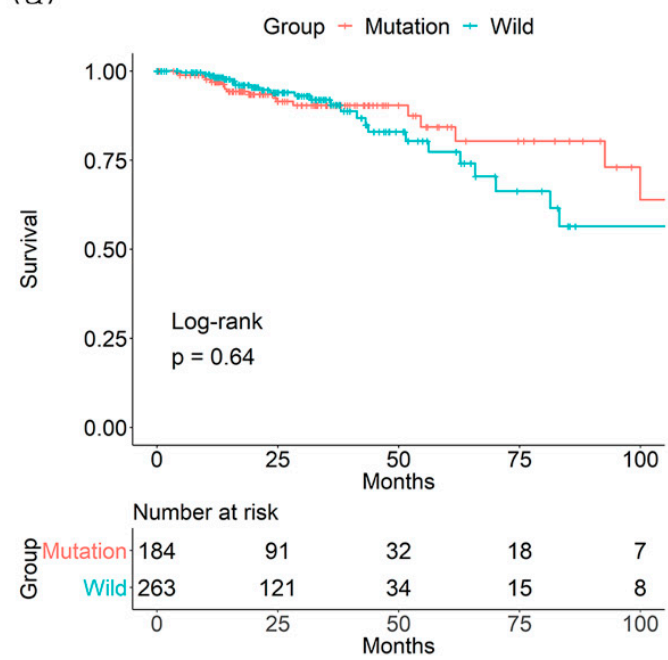

(b)

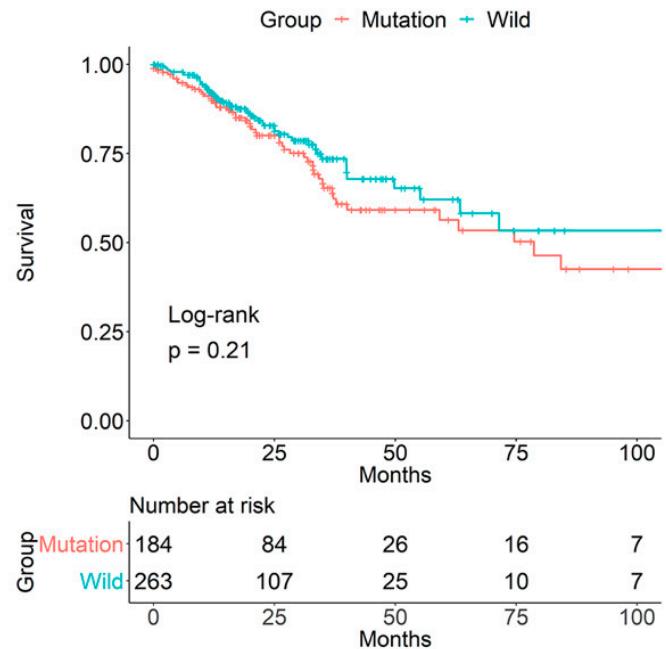

(c)

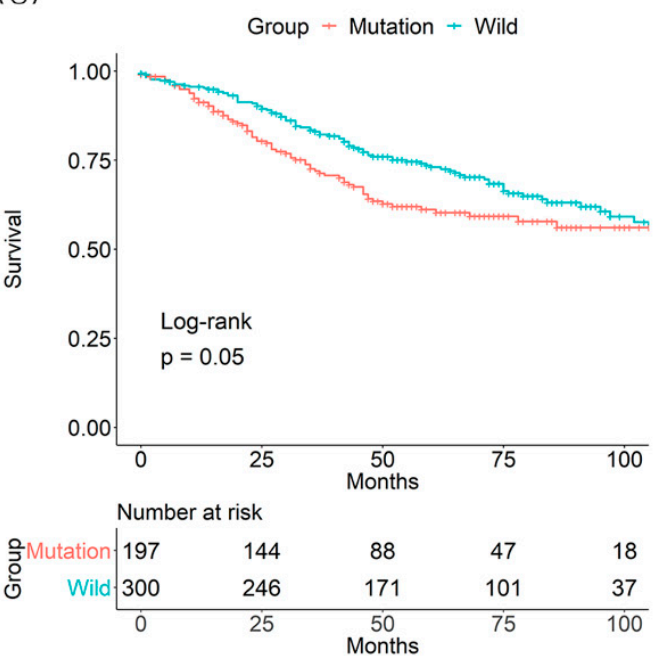

(d)

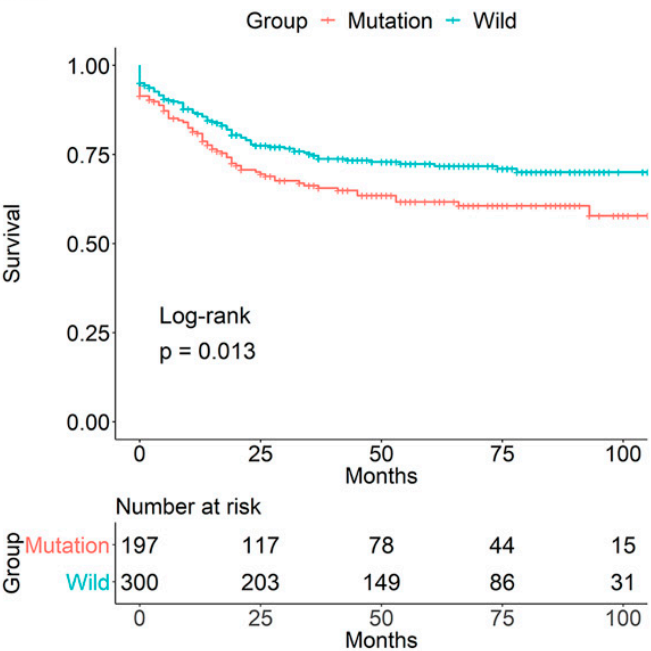

Figure 4. Effect of KRAS mutation on (a) 3-year overall survival and (b) 3-year disease-free survival from the The Cancer Genome Atlas (TCGA) database and on (c) 3-year overall survival and (d) 3-year disease-free survival from the GSE39582 database of colorectal cancer patients of all stages.

In the GSE39582 dataset, KRAS mutation positivity had a detrimental effect on 3-year OS rate (71.9\% mutation (+) vs. $83 \%$ mutation $(-), p=0.05$, number of patients: 116 vs. 214$)$ and DFS rate (66.3\% mutation (+) vs. $74.6 \%$ mutation $(-), p=0.013$, number of patients: 98 vs. 181) (Figure 4$)$. Among the CRC stages, stage III patients with KRAS mutation exhibited a lower 3-year OS (71.9\% mutation (+) vs. $89.4 \%$ mutation $(-), p=0.02$, number of patients: 41 vs. 71$)$ and DFS (54.0\% mutation $(+)$ vs. $73.5 \%$ mutation $(-), p=0.022$, number of patients: 31 vs. 59 ) rates (Figure 5). 
(a)

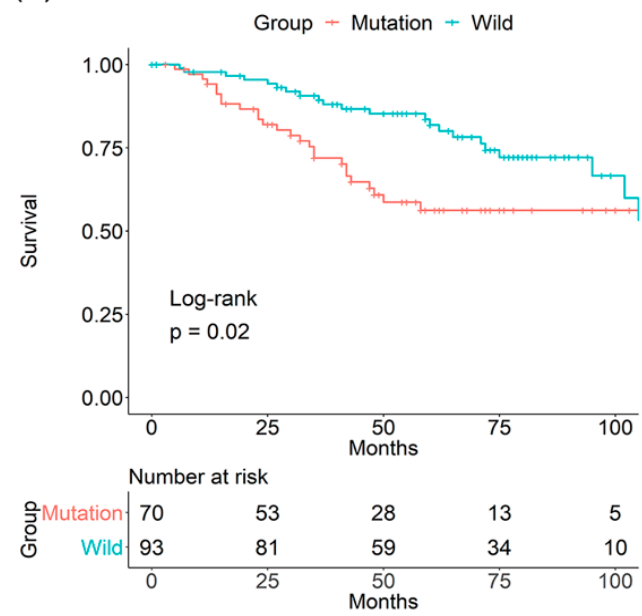

(b)

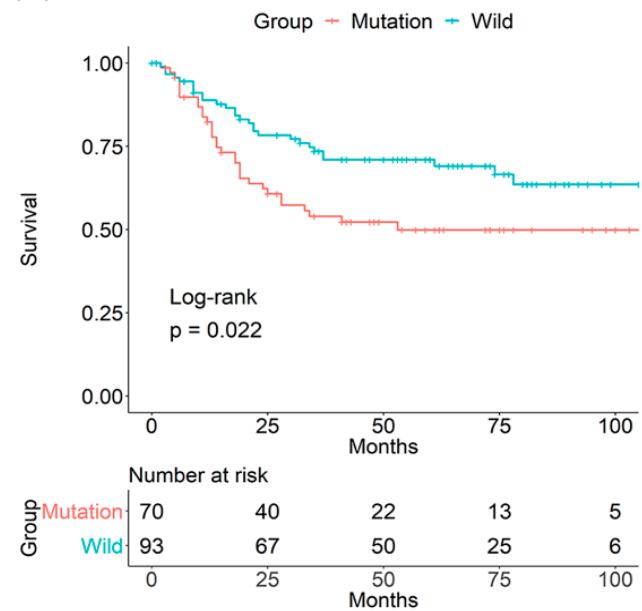

Figure 5. Effect of KRAS mutation on (a) 3-year overall survival and (b) 3-year disease-free survival from the GSE39582 database of colorectal cancer stage III patients.

\subsection{Combined Effect of BRAF and KRAS on Survival Outcome}

The 3-year OS rate of both BRAF and KRAS mutation (+) patients was $57.1 \%$, of both $B R A F$ and KRAS mutation (-) patients it was $88.5 \%$, of $B R A F$ mutation (-) and KRAS mutation (+) patients it was $92.1 \%$, and for BRAF mutation (+) and KRAS mutation (-) patients it was $100 \%(p<0.01$, number of patients: $2,59,11$, and 50 , respectively). There were no statistically significant differences regarding the 3-year DFS rate for the same gene combination ( $p=0.27$, number of patients: $2,46,9$, and 38 , respectively).

Further subgrouping by stage revealed a combined effect of $B R A F$ and KRAS mutation in stage II CRC patients only. Among the stage II CRC patients, the 3-year OS rate of both $B R A F$ and KRAS mutation (+) patients was $0 \%$, of BRAF mutation (-) and KRAS mutation (+) patients $96 \%$, of $B R A F$ mutation (+) and KRAS mutation (-) patients it was $100 \%$, and of both BRAF mutation (-) and KRAS mutation $(-) 100 \%$ ( $p<0.01$, number of patients: $0,33,7$, and 11 respectively). Among the stage II CRC patients, the 3-year DFS rate for both $B R A F$ and $K R A S$ mutation (+) patients was $25 \%$, for $B R A F$ mutation (-) and KRAS mutation (+) patients it was $76.3 \%$, for $B R A F$ mutation (+) and KRAS mutation (-) patients it was $83.1 \%$, and for both $B R A F$ and KRAS mutation $(-)$ patients it wasc $84.1 \%(p<0.01$, number of patients: $1,28,6$, and 17 , respectively). There were no statistically significant differences caused by the combined effect of BRAF and KRAS on CRC stages I, III, or IV, regarding 3-year OS ( $p=0.71,0.9$, and 0.91 , respectively) or DFS rates ( $p=0.85,0.44$, and 0.61 , respectively). 
There were no patients with both BRAF and KRAS mutations in the GSE39582 dataset.

\section{Discussion}

This study did not find a consistent association between the 3-year OS and DFS rates of CRC patients and $B R A F$ or KRAS mutation. Likewise, our subgroup analysis did not demonstrate consistent effect of $B R A F$ or KRAS mutations on OS and DFS according to each TNM stage. At present, the TNM staging system is the only classification routinely used in clinical practice to prognosticate CRC outcome [3]. However, the AJCC's 8th Edition of this classification system recommends BRAF and $K R A S$ for consideration in clinical care [3]. Accounting for $B R A F$ and KRAS applied concurrently with the TNM staging system was shown to improve the prognostic ability from a $0.61-0.68$ to $0.63-0.71$ concordance index (a performance measurement in survival analysis) for stage II and III CRC patients [21]. On the other hand, our results did not demonstrate a significant relationship between CRC outcome and BRAF or KRAS mutations. Although a decreased 3-year OS and DFS for KRAS-positive patients was observed in the GSE39582 database, its significance was circumscribed by the fact that the results were not reproduced by the counterpart TCGA database. One reason for the inconsistency is the discrepancy in the distribution of KRAS mutation between the two databases (384 (85.9\%) vs. 197 (39.6\%) for TCGA and GSE39582, respectively).

$B R A F$ is a serine/threonine kinase that contributes to cell proliferation, survival, and differentiation [22]. The glutamate for valine substitution at codon 600 in exon 15 (V600E) is the most common BRAF mutation, and is found in more than $90 \%$ of human malignancies such as papillary thyroid cancer, ovarian cancer, melanoma and CRC [22-24]. Around 4.7 to $20 \%$ of CRC patients have a BRAF mutation [22]. In a recent study of 1049 CRC patients with known BRAF status, $B R A F$ mutation had a detrimental effect on DFS and OS for stage I, II, and III CRC patients [25]. There is an association between $B R A F$ status and poorer clinical outcomes, especially in advanced stage CRC [26-28]. However, results in a pooled study of three trials (FOCUS, COIN, and PICCOLO), were inconsistent, showing worse OS but similar DFS for BRAF mutation (+) patients compared to the $B R A F$ mutation (-) counterparts [29]. Therefore, further research is required to elucidate the role of $B R A F$ in CRC.

The KRAS, a proto-oncogene, is a member of the Ras subfamily. It activates signaling cascades including the mitogen-activated protein kinase and PI3K pathways that regulate proliferation, motility, differentiation, and survival [30]. Mutations of KRAS are found in 30-50\% of CRCs and they are usually point mutations, primarily in codons 12 and 13 [31]. In metastatic CRC, KRAS mutation is predictive for anti-EGFR monoclonal antibodies such as cetuximab [32]. Although controversial, several meta-analyses reveal little prognostic value of KRAS mutations in CRC, in accordance with our results $[14,33]$ (Table 2).

Table 2. BRAF/KRAS status and prognosis in previous studies.

\begin{tabular}{|c|c|c|c|c|c|c|}
\hline Author, Year & Country & Time Frame & Patients & Stage & Outcomes & Poor Prognosis \\
\hline \multicolumn{7}{|l|}{$B R A F$} \\
\hline Won et al. 2017 & South Korea & 2010-2013 & 1049 & I, II, III & OS *, DFS ** & Mutant type \\
\hline Seligmann et al., 2017 & United Kingdom & $2000-2003$ & 231 & IV & PFS *** & Mutant type \\
\hline Roth et al. 2010 & Switzerland & $(-)$ & 1404 & II, III & $\mathrm{OS}^{*}, \mathrm{DFS}^{* *}$ & Mutant type \\
\hline \multicolumn{7}{|l|}{ KRAS } \\
\hline De Roock et al. 2010 & Belgium & 2001-2008 & 229 & IV & PFS *** & No difference \\
\hline Bokemeyer et al. 2011 & Germany & 2006-2007 & 315 & IV & PFS *** & Mutant type \\
\hline Schwartzberg et al. 2014 & Spain & 2009-2011 & 278 & IV & PFS $* * *, S^{*}$ & Mutant type \\
\hline
\end{tabular}

Co-existing BRAF and KRAS mutation are extremely rare because they are mutually exclusive [34]. In the unusual cases in which they do occur simultaneously, they seem to act synergistically to further shorten survival. In a study with $1000 \mathrm{CRC}$ patients with known BRAF and KRAS status, only 3 $(0.3 \%)$ harbored both mutations simultaneously and those patients fared significantly worse in terms 
of OS and DFS compared to their wild type counterpart [25]. In the GSE39582 dataset used in the present study, no patients had both mutations. Of the TCGA dataset, nine patients ( $2 \%$ ) out of 447 simultaneously had both BRAF and KRAS mutations. Their OS was shorter than those with other mutational combinations. We found no significant difference in DFS among these nine patients, but this may be attributed to the small sample size and lack of longitudinal data. Although the biologic mechanism through which the two mutually exclusive mutations co-exist is not clear, tumor heterogeneity is one theory. A sole malignancy consists of many sub-clone tumor cells, which may explain how two mutually exclusive $B R A F$ and $K R A S$ gene mutations can co-exist in a single tumor. The clinical significance of such a rare occurrence should be noted regarding the prognosis of the CRC and investigated further.

A limitation of our study was the brevity of patient survival information; in the TCGA and GSE39582 dataset it was insufficient in certain subgroups to comprehensively assess survival. Hence, we acquired data on the 3-year, instead of 5-year, survival rate. According to the surveillance, epidemiology, and end results data of 28,491 cases of CRC, an approximate 10-point reduction in OS rate was observed between the third and fifth post-operative year [35]. Three years was long enough to stratify the survival rates by the TNM stage. Therefore, the 3-year rate was deemed a sufficient surrogate indicator for our study. Furthermore, the quality of additional information on post-operative chemotherapy was poor. Although whether the patients received palliative or adjuvant therapy was mentioned, the specific regimen, total dosage, and the number of sessions administered was not mentioned, therefore rendering further qualitative analysis impossible.

In conclusion, we were unable to exclude the role of chance in the association between the mutational status of either BRAF or KRAS on the survival outcomes of CRC patients. The definitive role of $B R A F$ and $K R A S$ mutations on CRC survival with longer-term observation remains to be fully described in studies.

Author Contributions: Conceptualization, Methodology, Formal analysis, Writing-Original Draft Preparation, Visualization: J.-H.L., J.A., Y.J.C., and H.C. Validation, Investigation, Resources, Data curation, Writing-Review and Editing: W.S.P., E.K.C., E.K., R.S., S.C.H., S.J., and K.K. Supervision, Project Administration, Funding Acquisition: Y.J.C. and H.C.

Funding: This research was supported by the National Research Foundation of Korea (NRF) grant funded by the Korea government (Ministry of Science, ICT \& Future Planning, NRF-2016R1E1A1A01942072, http: //www.nrf.re.kr/index) and the Sookmyung Women's University Research Grants (1-1703-2033).

Conflicts of Interest: The authors have declared that no competing interests exist.

\section{References}

1. Jemal, A.; Bray, F.; Center, M.M.; Ferlay, J.; Ward, E.; Forman, D. Global cancer statistics. CA Cancer J. Clin. 2011, 61, 69-90. [CrossRef] [PubMed]

2. Brenner, H.; Kloor, M.; Pox, C.P. Colorectal cancer. Lancet 2014, 383, 1490-1502. [CrossRef]

3. Amin, M.B.; Edge, S.; Greene, F.; Byrd, D.R.; Brookland, R.K.; Washington, M.K.; Gershenwald, J.E.; Compton, C.C.; Hess, K.R.; Sullivan, D.C.; et al. AJCC Cancer Staging Manual, 8th ed.; Springer: New York, NY, USA, 2017; pp. XVII, 1032.

4. Huang, Z.; Duan, H.; Li, H. Identification of Gene Expression Pattern Related to Breast Cancer Survival Using Integrated TCGA Datasets and Genomic Tools. Biomed. Res. Int. 2015, 2015, 878546. [CrossRef] [PubMed]

5. Cancer Genome Atlas Research Network. Integrated genomic characterization of papillary thyroid carcinoma. Cell 2014, 159, 676-690. [CrossRef] [PubMed]

6. Devarakonda, S.; Morgensztern, D.; Govindan, R. Clinical applications of The Cancer Genome Atlas project (TCGA) for squamous cell lung carcinoma. Oncology 2013, 27, 899-906. [PubMed]

7. Lee, H.; Flaherty, P.; Ji, H.P. Systematic genomic identification of colorectal cancer genes delineating advanced from early clinical stage and metastasis. BMC Med. Genomics 2013, 6, 54. [CrossRef] [PubMed]

8. Markowitz, S.D.; Bertagnolli, M.M. Molecular origins of cancer: Molecular basis of colorectal cancer. N. Engl. J. Med. 2009, 361, 2449-2460. [CrossRef] 
9. Ogino, S.; Goel, A. Molecular classification and correlates in colorectal cancer. J. Mol. Diagn. 2008, 10, $13-27$. [CrossRef]

10. Passiglia, F.; Bronte, G.; Bazan, V.; Galvano, A.; Vincenzi, B.; Russo, A. Can KRAS and BRAF mutations limit the benefit of liver resection in metastatic colorectal cancer patients? A systematic review and meta-analysis. Crit. Rev. Oncol. Hematol. 2016, 99, 150-157. [CrossRef]

11. Sanz-Garcia, E.; Argiles, G.; Elez, E.; Tabernero, J. BRAF mutant colorectal cancer: Prognosis, treatment, and new perspectives. Ann. Oncol. 2017, 28, 2648-2657. [CrossRef]

12. Karagkounis, G.; Torbenson, M.S.; Daniel, H.D.; Azad, N.S.; Diaz, L.A., Jr.; Donehower, R.C.; Hirose, K.; Ahuja, N.; Pawlik, T.M.; Choti, M.A. Incidence and prognostic impact of KRAS and BRAF mutation in patients undergoing liver surgery for colorectal metastases. Cancer 2013, 119, 4137-4144. [CrossRef] [PubMed]

13. Schirripa, M.; Bergamo, F.; Cremolini, C.; Casagrande, M.; Lonardi, S.; Aprile, G.; Yang, D.; Marmorino, F.; Pasquini, G.; Sensi, E.; et al. BRAF and RAS mutations as prognostic factors in metastatic colorectal cancer patients undergoing liver resection. Br. J. Cancer 2015, 112, 1921-1928. [CrossRef] [PubMed]

14. Alwers, E.; Jia, M.; Kloor, M.; Blaker, H.; Brenner, H.; Hoffmeister, M. Associations Between Molecular Classifications of Colorectal Cancer and Patient Survival: A Systematic Review. Clin. Gastroenterol. Hepatol. 2018. [CrossRef] [PubMed]

15. Marisa, L.; de Reynies, A.; Duval, A.; Selves, J.; Gaub, M.P.; Vescovo, L.; Etienne-Grimaldi, M.C.; Schiappa, R.; Guenot, D.; Ayadi, M.; et al. Gene expression classification of colon cancer into molecular subtypes: Characterization, validation, and prognostic value. PLoS Med. 2013, 10, e1001453. [CrossRef] [PubMed]

16. Von Elm, E.; Altman, D.G.; Egger, M.; Pocock, S.J.; Gotzsche, P.C.; Vandenbroucke, J.P.; Initiative, S. The Strengthening the Reporting of Observational Studies in Epidemiology (STROBE) statement: Guidelines for reporting observational studies. J. Clin. Epidemiol. 2008, 61, 344-349. [CrossRef] [PubMed]

17. Cibulskis, K.; Lawrence, M.S.; Carter, S.L.; Sivachenko, A.; Jaffe, D.; Sougnez, C.; Gabriel, S.; Meyerson, M.; Lander, E.S.; Getz, G. Sensitive detection of somatic point mutations in impure and heterogeneous cancer samples. Nat. Biotechnol. 2013, 31, 213-219. [CrossRef]

18. Therneau, T.M. A Package for Survival Analysis in S. Available online: https://CRAN.R-project.org/ package $=$ survival (accessed on 26 November 2018).

19. Wickham, H. Ggplot2: Elegant Graphics for Data Analysis. Available online: https://cran.r-project.org/ web/packages/ggplot2/index.html (accessed on 25 October 2018).

20. R Core Team. R: A Language and Environment for Statistical Computing; 3.4.1; R Foundation for Statistical Computing: Vienna, Austria, 2016.

21. Dienstmann, R.; Mason, M.J.; Sinicrope, F.A.; Phipps, A.I.; Tejpar, S.; Nesbakken, A.; Danielsen, S.A.; Sveen, A.; Buchanan, D.D.; Clendenning, M.; et al. Prediction of overall survival in stage II and III colon cancer beyond TNM system: A retrospective, pooled biomarker study. Ann. Oncol. 2017, 28, 1023-1031. [CrossRef]

22. Jass, J.R. Classification of colorectal cancer based on correlation of clinical, morphological and molecular features. Histopathology 2007, 50, 113-130. [CrossRef]

23. De Sousa, E.M.F.; Wang, X.; Jansen, M.; Fessler, E.; Trinh, A.; de Rooij, L.P.; de Jong, J.H.; de Boer, O.J.; van Leersum, R.; Bijlsma, M.F.; et al. Poor-prognosis colon cancer is defined by a molecularly distinct subtype and develops from serrated precursor lesions. Nat. Med. 2013, 19, 614-618. [CrossRef]

24. Sadanandam, A.; Lyssiotis, C.A.; Homicsko, K.; Collisson, E.A.; Gibb, W.J.; Wullschleger, S.; Ostos, L.C.; Lannon, W.A.; Grotzinger, C.; Del Rio, M.; et al. A colorectal cancer classification system that associates cellular phenotype and responses to therapy. Nat. Med. 2013, 19, 619-625. [CrossRef]

25. Won, D.D.; Lee, J.I.; Lee, I.K.; Oh, S.T.; Jung, E.S.; Lee, S.H. The prognostic significance of KRAS and BRAF mutation status in Korean colorectal cancer patients. BMC Cancer 2017, 17, 403. [CrossRef]

26. Pietrantonio, F.; Petrelli, F.; Coinu, A.; Di Bartolomeo, M.; Borgonovo, K.; Maggi, C.; Cabiddu, M.; Iacovelli, R.; Bossi, I.; Lonati, V.; et al. Predictive role of BRAF mutations in patients with advanced colorectal cancer receiving cetuximab and panitumumab: A meta-analysis. Eur. J. Cancer 2015, 51, 587-594. [CrossRef] [PubMed]

27. Rowland, A.; Dias, M.M.; Wiese, M.D.; Kichenadasse, G.; McKinnon, R.A.; Karapetis, C.S.; Sorich, M.J. Meta-analysis of BRAF mutation as a predictive biomarker of benefit from anti-EGFR monoclonal antibody therapy for RAS wild-type metastatic colorectal cancer. Br. J. Cancer 2015, 112, 1888-1894. [CrossRef] [PubMed] 
28. Chen, D.; Huang, J.F.; Liu, K.; Zhang, L.Q.; Yang, Z.; Chuai, Z.R.; Wang, Y.X.; Shi, D.C.; Huang, Q.; Fu, W.L. BRAFV600E mutation and its association with clinicopathological features of colorectal cancer: A systematic review and meta-analysis. PLoS ONE 2014, 9, e90607. [CrossRef] [PubMed]

29. Seligmann, J.F.; Fisher, D.; Smith, C.G.; Richman, S.D.; Elliott, F.; Brown, S.; Adams, R.; Maughan, T.; Quirke, P.; Cheadle, J.; et al. Investigating the poor outcomes of BRAF-mutant advanced colorectal cancer: Analysis from 2530 patients in randomised clinical trials. Ann. Oncol. 2017, 28, 562-568. [CrossRef] [PubMed]

30. Poulogiannis, G.; Luo, F.; Arends, M.J. RAS signalling in the colorectum in health and disease. Cell Commun. Adhes. 2012, 19, 1-9. [CrossRef] [PubMed]

31. Arrington, A.K.; Heinrich, E.L.; Lee, W.; Duldulao, M.; Patel, S.; Sanchez, J.; Garcia-Aguilar, J.; Kim, J. Prognostic and predictive roles of KRAS mutation in colorectal cancer. Int. J. Mol. Sci. 2012, 13, 12153-12168. [CrossRef]

32. Tran, N.H.; Cavalcante, L.L.; Lubner, S.J.; Mulkerin, D.L.; LoConte, N.K.; Clipson, L.; Matkowskyj, K.A.; Deming, D.A. Precision medicine in colorectal cancer: The molecular profile alters treatment strategies. Ther. Adv. Med. Oncol. 2015, 7, 252-262. [CrossRef]

33. Shan, L.; Li, M.; Ma, J.; Zhang, H. PCR-based assays versus direct sequencing for evaluating the effect of KRAS status on anti-EGFR treatment response in colorectal cancer patients: A systematic review and meta-analysis. PLoS ONE 2014, 9, e107926. [CrossRef]

34. Morkel, M.; Riemer, P.; Blaker, H.; Sers, C. Similar but different: Distinct roles for KRAS and BRAF oncogenes in colorectal cancer development and therapy resistance. Oncotarget 2015, 6, 20785-20800. [CrossRef]

35. Edge, S.; Byrd, D.; Compton, C.; Fritz, A.; Greene, F.; Trotti, A. AJCC Cancer Staging Manual, 7th ed.; Springer: New York, NY, USA, 2009.

(C) 2019 by the authors. Licensee MDPI, Basel, Switzerland. This article is an open access article distributed under the terms and conditions of the Creative Commons Attribution (CC BY) license (http://creativecommons.org/licenses/by/4.0/). 